\title{
Numerical Analysis of a Friedlander Localized Blast Load on a Rigid Roadway Pavement Using Levy's Problem
}

\author{
Sofia W Alisjahbana ${ }^{\mathrm{a},{ }^{*}}$, Ade Asmi ${ }^{\mathrm{a}, 1}$, Safrilah ${ }^{\mathrm{a}, 2}$, Jouvan Chandra Pratama ${ }^{\mathrm{a}, 3}$, Buntara S. Gan ${ }^{\mathrm{b}}$, \\ Irene Alisjahbana ${ }^{\mathrm{c}}$ \\ ${ }^{a}$ Civil Engineering Department, Bakrie University, Kuningan Jakarta, Jakarta Selatan, 12920, Indonesia \\ E-mail: * sofia.alisjahbana@bakrie.ac.id (corresponding author); ${ }^{1}$ ade.asmi@bakrie.ac.id, ${ }^{2}$ safrilah@bakrie.ac.id, \\ jouvan.chandra@bakrie.ac.id \\ ${ }^{b}$ Department of Architecture, College of Engineering, Nihon University, Chiyoda City, Tokyo, 101-0061, Japan \\ E-mail: buntara@arch.ce.nihon-u.ac.jp \\ ${ }^{c}$ Department of Civil and Environmental Engineering, Stanford University, 450 Jane Stanford Way, Stanford, CA 94305-2004, USA \\ E-mail: alisjahbana@stanford.edu
}

\begin{abstract}
The increasing number of terrorist attacks in recent years have shown that in designing structures such as floor slabs and rigid roadway pavement, the effect of blast loads should be taken into consideration. In this study, we analyze the blasting effect on the rigid roadway pavements and provide a numerical example of a toll road shoulder section exposed to this blast load. We assumed that the rigid roadway slab is connected by dowels and tie-bars along its edges. Furthermore, we modeled the blast as a Friedlander blast load, where it is initially a positive pressure before decreasing to a negative pressure due to drag. We obtained the vertical shear force that is carried by the dowels and tie-bars along its edges, the time history of the rigid roadway slab deflection, and identified the parameters of the sub-grade that has significant effects on the dynamic response of the rigid roadway pavement. We carried out the numerical analysis using the first and the second type of Levy's problem to find the Eigenvalues and Eigenvectors and to predict the maximum deformed shape, the maximum vertical shear forces along the joint of the rigid roadway pavement subjected to the blast load. Our results show that the duration ratio of the blast loading, the thickness of the slab, and the stiffness coefficient of the soil has a significant influence on the dynamic response of the slab. This paper provides essential techniques in increasing the capacity of a rigid roadway slab against explosive effects.
\end{abstract}

Keywords — rigid roadway pavement; friedlander blast load; levy's problem; dynamic response.

\section{INTRODUCTION}

Orthotropic slabs are one of the most vital structural elements in construction. Almost every project, whether it be in toll roads, buildings, schools, apartments, malls, shelters, and other public facilities, contain slabs subjected to various condition and environment. Due to this, slabs should be evaluated at different conditions and situations to achieve a functional and economical design that is safe. To satisfy both strength and serviceability requirements, slab responses that should be analyzed include deflections, internal shear force, and flexural stresses.

The types of load commonly modeled in roadway slab analysis include gravity load and traffic loads. However, many engineers ignore the effects of other dynamic loads, particularly blast loads. Blast loads, or explosions, are defined as the rapid release of energy as a mass that is converted to regions of extremely high strain rates. The high strain rates would change the dynamic mechanical properties of the structure and potentially cause damage to the structural elements [1]. Given the potential consequence of blast loads, an effective slab design should, therefore, anticipate any load that may potentially affect the slab, including blast loads, which have unique characteristics and effects on slabs that must be accounted for.

Until now, most researchers have focused on the problems of traffic load-roadway interaction but not of the blast loadroadway interaction. Meanwhile, because modern terrorism has increased in recent years, the risk of blast loads on roadway structures has also increased. Developed societies have become very dependent on complex and vulnerable systems such as toll roads, railways, runways, large 
shopping areas, hotels, and business centers enabling the terrorists to have many suitable targets. Explosive devices have become smaller in size and more powerful over the years, further increasing the risk of blast loads on roadway structures [2].

It is therefore essential to develop a procedure that will give practical design solutions for the design of new or retrofitted roadway structures to be able to withstand the effects of explosive loads. In designing new or retrofitted roadway structures, engineers must calculate the acting forces of certain blast scenarios, including the type and weight of the used explosive, as well as the distance from the blast load source to the roadway structure. These forces would then be applied to the structural system to design the roadway sections and connections, thus ensuring sufficient robustness of the roadway slab to survive the effects of the computed actions [3].

In 2018, Alisjahbana et al [4] investigated the dynamic response of an orthotropic concrete plate subjected to the positive and negative phases of blast loading and found that negative phases of the localized blast load an important factor in the dynamic response of the stiffened orthotropic plates. In their study, they found that parameters such as stiffness configuration, location of load, and plate thickness, affect the dynamic response of plates subject to Friedlander blast loads. They focused their study on the evaluation of midpoint displacements and maximum stress distribution within the plate region.

Since the dynamic response of the rigid roadway pavement subjected to the localized blast load has not previously been studied in detail, this paper gives an overview of the dynamic response of the rigid roadway slab to a localized blast load by using the first and the second Levy's problems. The first and second Levy's problem has been shown to provide accurate results, especially in calculations of high modes [5]. We focus our analysis on the the midspan due to soil condition above the slab, and the vertical shear force distribution along the edges of the slab to provide protection of the roadway against blast load effects.

\section{MATERIAL AND METHOD}

\section{A. Rigid Roadway Slab and Blast Load Modeling}

In this study, we consider the model of a rigid roadway pavement supported by a two-parameter soil foundation. Two-parameter soil foundations are commonly used to model soil and accounts for shear deformations [6]. The equation of motion for a rigid roadway slab is derived based on the elastic thin slabs theory and Newton II theory as described by [7]:

$$
\begin{aligned}
& D_{x} \frac{\partial W(x, y)}{\partial x^{4}} T(t)+2 B \frac{\partial^{4} W(x, y)}{\partial x^{2} \partial y^{2}} T(t)+D_{y} \frac{\partial^{4} W(x, y)}{\partial y^{4}} T(t)+\xi h W(x, y) \frac{d T(t)}{d t} \\
& +\rho h W(x, y) \frac{d^{2} T(t)}{d t^{2}}+k_{f} W(x, y) T(t)-G_{s} \nabla^{2} W(x, y) T(t)=p(x, y, t)
\end{aligned}
$$

According to Equation (1), $W(x, y)$ is the spatial function, $T(t)$ is the temporal function, $D_{x}$ is the flexural rigidity of slab in the $x$ direction, $B$ is the torsional rigidity, $D_{y}$ is the flexural rigidity of slab in the $y$ direction, $\xi$ is the damping ratio, $\rho$ is the mass density, $k_{f}$ is the stiffness of the soil, $G_{s}$ is the shear modulus of the soil, $\nabla^{2}$ is the Laplacian operator and $p(x, y, t)$ is the load's function.

The solution of equation of motion of the slab according to Equation (1) can be found by using the method of separation of variables which consists of the multiplication of the spatial function $W(x, y)$ and the temporal function $T(t)$. The function $W(x, y)$ is determined from the solutions of the first and the second equations of the Levy type problems.

A few studies have developed the equation for a localized Friedlander load for both the positive phase and negative phase of the load. The positive phase of a blast load

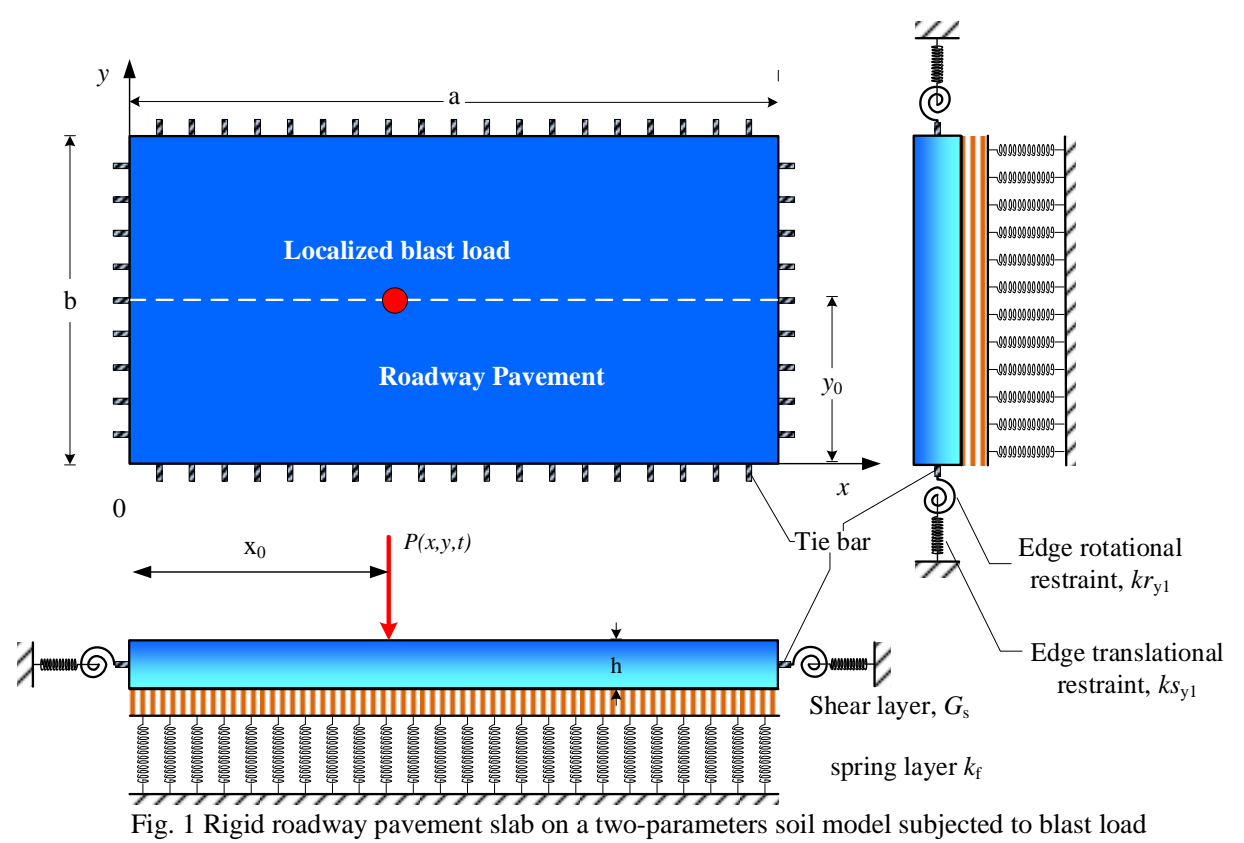

method used to model the blast loading on rigid roadway slab, including the shoulder section, vertical deformation at is commonly expressed as a linear or exponential function and acts in the direction towards the slab. In this case, we 
utilized an exponential function for the positive phase [8] and the cubic function for the negative phase as follows [9]:

$$
P(t)=P_{\max }\left(1-\frac{t}{t d p}\right) e^{-\alpha \frac{t}{t d p}} \text { for } 0 \leq \mathrm{t} \leq t d p
$$

and

$$
P(t)=-P_{\min }\left(\frac{6.75(t-t d p)}{t d n}\right)\left(1-\frac{(t-t d p)}{t d n}\right)^{2}
$$

$$
\text { for } t d p \leq t \leq(t d p+t d n)
$$

According to Equation (3) after the positive phase $(0 \leq t$ $\leq t d p)$ is completed, the load enters its negative phase ( $t d p \leq$ $t \leq t d p+t d n)$, changing into vacuum force. Generally, this phase has an amplitude smaller than the positive phase, but it has a much longer duration, approximately twice the length of the positive phase. The function of a Friedlander load for both the positive and negative phase is shown in Fig. 2 .

The position of the blast load at any time $t$ can be expressed by using the Dirac delta function [7]:

$$
p(x, y, t)=P(t) \delta\left[x-x_{0}\right] \delta\left[y-y_{0}\right]
$$

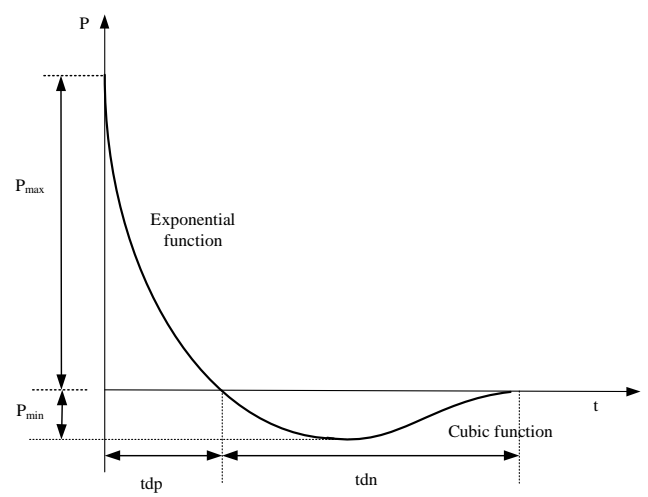

Fig. 2 Friedlander blast load described by exponential function during the positive phase $(t d p)$ and cubic function during the negative phase $(t d n)$

To solve the equation of motion of roadway slab according to Equation (1), the following boundary conditions along the edges of rigid roadway slab are needed:

In the $x$ direction:

$V_{x}=-D_{x}\left(\frac{\partial^{3} W(x, y) T(t)}{\partial x^{3}}+\left(\frac{4 D_{t r}}{D_{x}}+v_{y}\right) \frac{\partial^{3} W(x, y) T(t)}{\partial x \partial y^{2}}\right)=k s_{x} W(x, y) T(t)$

$M_{x}=-D_{x}\left(\frac{\partial^{2} W(x, y) T(t)}{\partial x^{2}}+v_{y} \frac{\partial^{2} W(x, y) T(t)}{\partial y^{2}}\right)=k r_{x} \frac{\partial W(x, y) T(t)}{\partial x}$

In the y direction:

$$
\begin{gathered}
V_{y}=-D_{y}\left(\frac{\partial^{3} W(x, y) T(t)}{\partial y^{3}}+\left(\frac{4 D_{t r}}{D_{y}}+v_{x}\right) \frac{\partial^{3} W(x, y) T(t)}{\partial x^{2} \partial y}\right)=k s_{y} W(x, y) T(t) \\
M_{y}=-D_{y}\left(\frac{\partial^{2} W(x, y) T(t)}{\partial y^{2}}+v_{x} \frac{\partial^{2} W(x, y) T(t)}{\partial x^{2}}\right)=k r_{y} \frac{\partial W(x, y) T(t)}{\partial y} \\
D_{t r}=\frac{\left(1-\sqrt{v_{x} V_{y}}\right) \sqrt{D_{x} D_{y}}}{2}
\end{gathered}
$$

The coefficient vertical translational restriction and the coefficient rotation along the edges of the slab due to the existence of dowels and tie bars along the $x$-axis and $y$-axis are characterized by $k s_{x}, k r_{x}, k s_{y}, k r_{y}$ respectively [10].

In this study, we include the shoulder part of the slab where the blast load was assumed to have exploded.

\section{B. Solution for Levy's Problem}

In this part of the paper, we show the free vibration response of orthotropic rigid roadway slab with boundary conditions according to Equations (5) to (9) resting on a two parameters soil foundation model. The natural period of the system and the eigen vectors in the $\mathrm{x}$ and $\mathrm{y}$ directions were determined through the Levy's problem [5].

We obtained the eigenvalue for a roadway slab with dowels and tie-bars support on all sides by solving the equation in a manner similar to a simply supported plate. We substituted the integer index values for the $x$ and $y$ directions with coefficients $p$ and $q$ for the mode shapes in the $x$ and $y$ directions respectively. The natural period $(T n)$ for a roadway slab with dowel and tie-bars supports can then be expressed as Equation (10):

$T n=2 \pi \sqrt{\rho h}\left(D_{x} \frac{p^{4} \pi^{4}}{a^{4}}+2 B \frac{p^{2} q^{2} \pi^{4}}{a^{2} b^{2}}+D_{y} \frac{q^{4} \pi^{4}}{b^{4}}+k_{f}+G_{s}\left(\frac{p^{2} \pi^{2}}{a^{2}}+\frac{q^{2} \pi^{2}}{b^{2}}\right)\right)^{-0.5}$

The values of $p$ and $q$ in Equation (10) are the real roots of transcendental equations, which we determined from the determinant of two matrices of the imposed boundary conditions for both the $x$ and $y$ directions [8]. Because we solved the auxiliary problems for each direction separately and assumed the perpendicular direction vibrates harmonically, this problem was categorized as a Levy type problem. Each of the $x$ and $y$ directions had a Levy type problem called the first auxiliary and second auxiliary equations. The first auxiliary equation provided the solution for the position function $X(x)$ in the $x$ direction while the $y$ direction vibrated harmonically. On the other hand, the second auxiliary equation provided the solution for the position function $Y(y)$ in the $y$ direction while the $x$ direction vibrated harmonically.

We obtained the solution for the first auxiliary equation in the $x$ direction by utilizing the following trial function:

$$
W(x, y)=X(x) \sin \frac{q \pi y}{b}
$$

where $X(x)$ is the position function from the orthotropic plate in the $\mathrm{x}$ direction.

The solution of the first auxiliary equation was expressed as [12]:

$$
\begin{aligned}
X(x) & =A_{1} \cosh \frac{\sqrt{\frac{2 B q^{2} a^{2}}{D_{x}}+p^{2} b^{2}+\frac{G_{s} a^{2} b^{2}}{\pi^{2} D_{x}}}}{a b} \pi x+A_{2} \sinh \frac{\sqrt{\frac{2 B q^{2} a^{2}}{D_{x}}+p^{2} b^{2}+\frac{G_{s} a^{2} b^{2}}{\pi^{2} D_{x}}}}{a b} \pi x \\
& +A_{3} \cos \frac{p \pi x}{a}+A_{4} \sin \frac{p \pi x}{a}
\end{aligned}
$$

Based on Equations (5) and (6), $A_{1}, \ldots ., A_{4}$ are constants which were determined using the boundary conditions along the edges. Applying the boundary conditions along $x=0$ and $x=a$, resulted in the linear algebra equation in terms of $A_{i}$, as follows:

$$
\left[\begin{array}{llll}
\alpha_{11} & \alpha_{12} & \alpha_{13} & \alpha_{14} \\
\alpha_{21} & \alpha_{22} & \alpha_{23} & \alpha_{24} \\
\alpha_{31} & \alpha_{32} & \alpha_{33} & \alpha_{34} \\
\alpha_{41} & \alpha_{42} & \alpha_{43} & \alpha_{44}
\end{array}\right]\left\{\begin{array}{l}
A_{1} \\
A_{2} \\
A_{3} \\
A_{4}
\end{array}\right\}=\left\{\begin{array}{l}
0 \\
0 \\
0 \\
0
\end{array}\right\}
$$

where the coefficients $\alpha_{i j}$ are obtained by setting the determinant of Equation (13) to equal to zero for a nontrivial condition. [4]:

The spatial function in the $y$ direction can be expressed by 


$$
\begin{aligned}
Y(y)= & B_{1} \cosh \frac{\sqrt{\frac{2 B p^{2} b^{2}}{D_{y}}+q^{2} a^{2}+\frac{G_{s} a^{2} b^{2}}{\pi^{2} D_{y}}}}{a b} \pi y+B_{2} \sinh \frac{\sqrt{\frac{2 B p^{2} b^{2}}{D_{y}}+q^{2} a^{2}+\frac{G_{s} a^{2} b^{2}}{\pi^{2} D_{y}}}}{a b} \pi y \\
& +B_{3} \cos \left(\frac{q \pi y}{b}\right)+B_{4} \sin \left(\frac{q \pi y}{b}\right)
\end{aligned}
$$

We solved the second auxiliary in the y direction with a procedure analogous to the above derivations. By applying the boundary conditions along the shoulder section of the road slab at $y=0$ and $y=\mathrm{b}$ according to Equations (7) and (8), we obtained the auxiliary matrix in the y direction as follows:

$$
\left[\begin{array}{cccc}
\beta_{11} & \beta_{12} & \beta_{13} & \beta_{14} \\
\beta_{21} & \beta_{22} & \beta_{23} & \beta_{24} \\
\beta_{31} & \beta_{32} & \beta_{33} & \beta_{34} \\
\beta_{41} & \beta_{42} & \beta_{43} & \beta_{44}
\end{array}\right]\left\{\begin{array}{l}
B_{1} \\
B_{2} \\
B_{3} \\
B_{4}
\end{array}\right\}=\left\{\begin{array}{l}
0 \\
0 \\
0 \\
0
\end{array}\right\}
$$

where the coefficients $\beta_{i j}$ are obtained by setting the determinant of Equation (15) to equal to zero for a nontrivial condition.

The total dynamic response of the slab subjected to a localized transversal blast load requires two distinct solutions, the homogeneous solution and the forced vibration solution. The homogeneous solution is the solution for the structure that was imposed by an initial deformation or initial velocity condition that causes the structure to vibrate freely. In order to solve for the homogeneous solution, we set the right side of the governing equation of motion according to Equation (1) to equal to zero. As explained previously, this was solved through the separation of variables method. This method simplified the above equations by separating the governing differential equation into two different equations: the spatial differential equation $W(x, y)$ and the temporal differential equation $T(t)$. The spatial differential equation is a function of position $x$ and $y$ while the temporal differential equation is a function of time $t$. The homogeneous solution was expressed as the following equation:

$$
w_{h}(x, y, t)=W(x, y) e^{-\xi \omega t}\left(C_{1} \cos \omega \sqrt{1-\xi^{2}} t+C_{2} \sin \omega \sqrt{1-\xi^{2}} t\right)
$$

Similar to the method for solving the homogeneous solution, we also solved the forced vibration solution using the separation of variables method. The forced vibration solution has initial conditions cause by the transient vibration without loading and is expressed as follows:

$$
w_{p}(x, y, t)=\frac{W(x, y) X\left(x_{0}\right) Y\left(y_{0}\right)}{\int_{x=0}^{a} \int_{y=0}^{b} X^{2}(x) Y^{2}(y) d x d y}\left(\frac{1}{\rho h \omega \sqrt{1-\xi^{2}}}\right) \int_{0}^{1}\left[P(\tau)\left(e^{-\xi \rho(t-\tau)}\right) \sin \left(\omega \sqrt{1-\xi^{2}}(t-\tau)\right)\right] d \tau
$$

Thus, we obtained the general solution of the associated rigid roadway pavement subjected to localized blast load by adding the homogeneous solution according to Equation (16) and the forced vibration solution according to Equation (17).

\section{RESULTS AND DISCUSSION}

We used the Mathematica-Wolfram package version 12.1 to solve the dynamic responses of the roadway slab, which included integrating the Duhamel formula to find the temporal function $T(t)$.
In this study, we studied one segment of the rigid roadway pavement $\left(5.0 \times 3.5 \mathrm{~m}^{2}\right)$ with dowel and tie bar connections along three edges of the slab and one side with shoulder edge condition. This slab was subjected to the localized blast load. We used $0.25 \mathrm{~m}, 0.26 \mathrm{~m}$ and $0.27 \mathrm{~m}$ as the slab thickness in the numerical studies.

The adopted material properties were: Young's modulus, $E_{x}=27.8 \times 109 \mathrm{Nm}^{-2}, E_{y}=30.0 \times 109 \mathrm{Nm}^{-2}$, Poisson coefficients, $v_{x}=0.18, v_{y}=0.15, \xi=5 \%$ and mass density $\rho=2400 \mathrm{kgm}^{-3}$. The coefficients restriction along the edge of the slab were: $k s_{x 1}, k s_{x 2}=2.825 \times 10^{7} \mathrm{Nm}^{-1}, k s_{y 1}=0, k s_{y 2}=5.488 \times 10^{7} \mathrm{Nm}^{-1}$, $k r_{x 1}, k r_{x 2}, k r_{y 1}, k r_{y 2}=10^{6} \mathrm{Nm} \operatorname{rad}^{-1}$. The values of two parameters soil beneath the roadway slab were $k_{f}=2.725 \times 10^{7}$ $\mathrm{Nm}^{-1}$ and $G s=9.52 \times 10^{6} \mathrm{Nm}^{-1}$.

In this study, we used a localized blast load developed by Friedlander, with the positive phase using an exponential function and the negative phase using the cubic negative phase developed by Ganström [13]. Rigby et al [14] showed that for the negative phase of a Friedlander load, the cubic negative phase approach yields the most accurate results. The parameters used for the Friedlander load are identical to the ones used in studies by Susler et al [9] and Draganic, H. and Sigmund, V. [15]. The parameters are tabulated in Table 1 .

TABLE I

FRIEDLANDER LOAD ARAMETERS

\begin{tabular}{|l|l|l|}
\multicolumn{2}{|l}{ FRIEDLANDER LOAD ARAMETERS } \\
\hline Symbol & & Value and units \\
\hline$P_{\max }$ & $\begin{array}{l}\text { Positive phase } \\
\text { amplitude }\end{array}$ & $2.89 \times 10^{4}\left[\mathrm{Nm}^{-2}\right]$ \\
\hline$P_{\min }$ & $\begin{array}{l}\text { Negative phase } \\
\text { amplitude }\end{array}$ & $7.23 \times 10^{3}\left[\mathrm{Nm}^{-2}\right]$ \\
\hline$t_{0}$ & Initial load time & $0[\mathrm{~s}]$ \\
\hline$t d p$ & $\begin{array}{l}\text { Duration of } \\
\text { positive phase }\end{array}$ & $0.0018[\mathrm{~s}]$ \\
\hline$t d n$ & $\begin{array}{l}\text { Duration of } \\
\text { negative phase }\end{array}$ & 0.0036 \\
\hline$\alpha$ & $\begin{array}{l}\text { Wave shape } \\
\text { coefficient }\end{array}$ & 0.35 \\
\hline
\end{tabular}

In the first part of the numerical results, we focused on the dynamic response of the roadway slab, which included the vertical shear force distribution along the edges and the maximum flexural stress of the system, as an effect to the duration of the positive phase of the blast load $(t d p)$. In the remainder of this paper, we demonstrate the influence of the stiffness of the soil foundation model and the slab thickness to the total response of the system subjected to a localized blast load.

\section{A. Effect of the Duration of the Positive Phase (tdp)}

To demonstrate the influence of the duration of the positive phase on the dynamic response of the roadway slab, we solved the equation of motion by using the method of separation of variables and the two Levy's problems under the localized blast load parameter taken from Susler et al [9]. The variation of the duration of positive phase showed an important influence on the response of the roadway slab. The maximum dynamic deflections for all variation of the $t d p$ are shown in Fig. 3. 


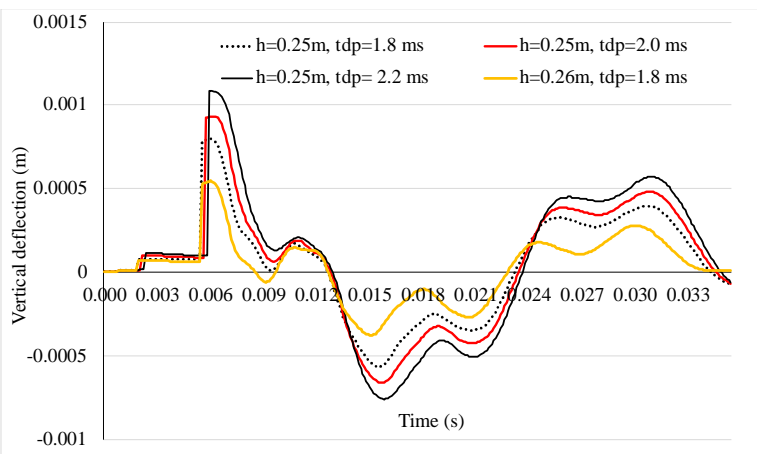

Fig. 3 Influence of tdp to the vertical displacement of the slab computed at the mid-span.

Our results show that the duration of positive phase has the important role in increasing the mid-point deflection of the roadway slab. As expected, increasing the duration of positive phase by $0.2 \mathrm{~ms}$ results in an increase of the maximum dynamic deflection by $17.2 \%$, which occurs right after the negative phase of the blast load applied at the system. Vertical shear force distribution along the edges of the road-way slab at $x=a$ can be seen in Fig. 4.

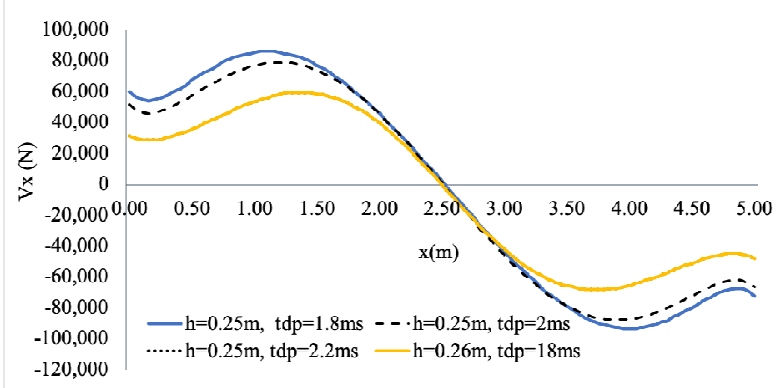

Fig. 4 Vertical shear force distribution along the edge of the roadway slab $(\mathrm{x}=\mathrm{a})$ computed at free vibration phase $(\mathrm{t}=5.5 \mathrm{~ms})$.
Fig. 4 shows the distribution of vertical shear force $(V x)$ along the edge of the roadway slab where the dowels exists. This was computed at $t=5.5 \mathrm{~ms}$ when the free vibration phase occurred for different values of $t d p$. Referring to Fig. 4, it is clear that the maximum vertical shear force occurs not along the edge of the slab, but at about a quarter of the slab for all the value of $t d p$ considered in this paper.

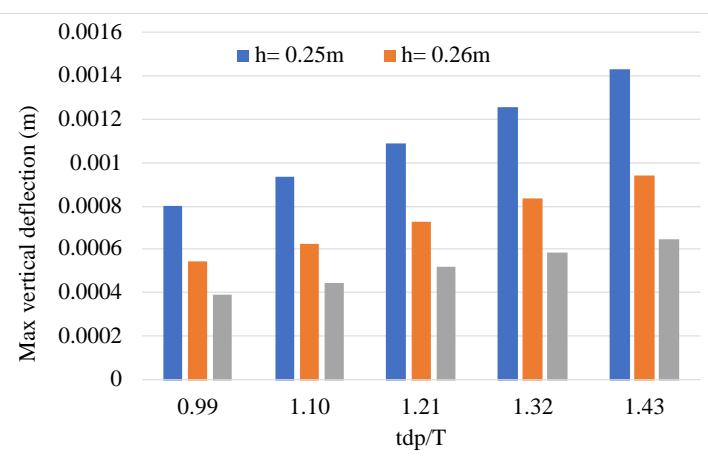

Fig. 5 Maximum vertical displacement versus $t d p / T n$ computed at mid-span of the rigid roadway slab for different values of $h$.

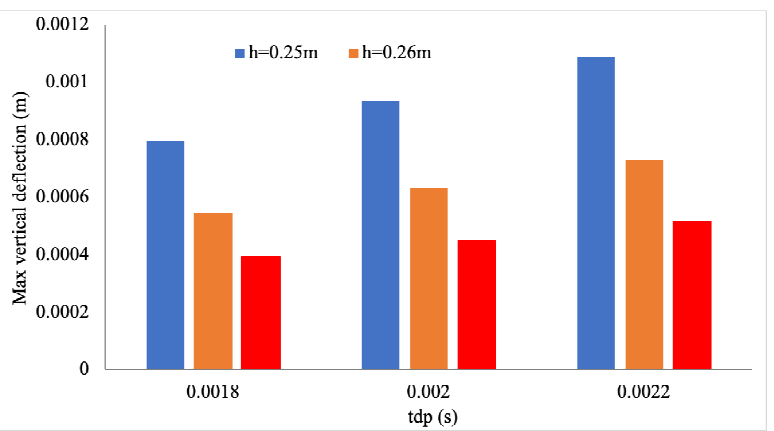

Fig. 6 Maximum flexural stress versus tdp subjected to a localized blast load for different values of thickness.

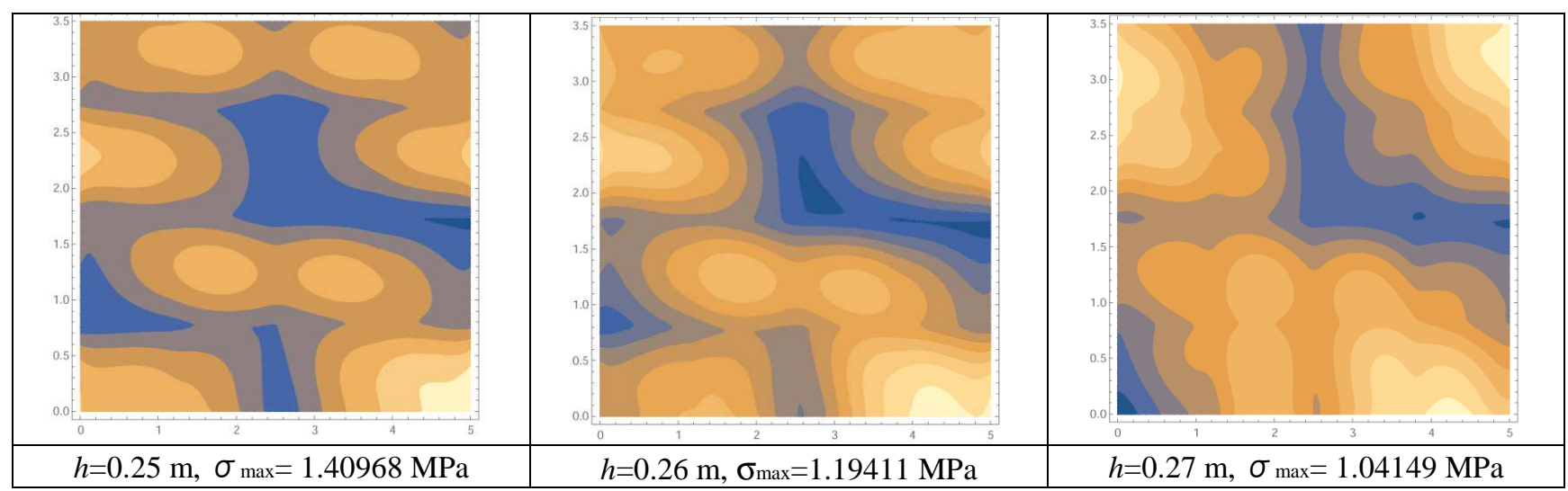

Fig. 7 Flexural stresses distribution of the rigid roadway slab subjected to a localized blast load computed at $t=5.5 \mathrm{~ms}$.

\section{B. Effect of the Thickness of Roadway Slab}

To demonstrate the influence of the slab thickness, we solved the maximum vertical deflections of the system using three-thickness values, which are $h=0.25 \mathrm{~m}, h=0.26 \mathrm{~m}$ and $h=0.27 \mathrm{~m}$, respectively. By increasing the thickness of the slab by $0.01 \mathrm{~m}$, the maximum vertical deflection of the system decreases by $31.6 \%$ for the value of $t d p=18 \mathrm{~ms}$. Travakoli and Kiakojouri [16] has shown that blast loads produce very high strain rates $\left(10^{2}-10^{4} \mathrm{~s}^{-1}\right)$. According to this, high loading rates would alter the response of the system. This fact is further confirmed by the numerical study shown in Fig. 5. The system will resist a longer $t d p$ as long as the thickness of the plate is thick enough. For the same value of $P_{\max }$ applied at the system, the maximum vertical deflection of the system with $t d p / T n=0.99$ and $h=0.25 \mathrm{~m}$ was $7.976 .10^{-4} \mathrm{~m}$, while the system with $h=0.26 \mathrm{~m}$ had a maximum vertical displacement of $7.276 .10^{-4} \mathrm{~m}$. For this 
study, the flexural stresses calculated were the maximum and the minimum stresses, while the maximum shear stresses were based on the Mohr's theory.

In order to find those maximum stresses, we calculated the stress components $\sigma_{x}, \sigma_{y}$, and $\tau_{x y}$ using equations that show the relationship between displacements and stress components [8]. For all stress calculations, we used blast loads located at the midspan of the slab. The plot showing the resulting maximum flexural stress versus $t d p$ is illustrated in Fig. 6. For all types of stresses evaluated, the roadway slab with an additional $0.01 \mathrm{~m}$ of slab thickness provided the greatest reduction in stresses as shown in Fig. 6 and Fig. 7. The percentage of reductions in stresses from adding the values of an additional $0.01 \mathrm{~m}$ of slab thickness is $15.29 \%$

Our results show that if the main design purpose of the slab was to reduce the dynamic deflection, the vertical shear force, and the maximum flexural stress along the edge of the plate, the slab thickness should be increased.

\section{Effect of the soil stiffness $\left(k_{f}\right)$}

We analyzed the influence of soil stiffness $\left(k_{f}\right)$ between the intervals $27.5 \mathrm{MNm}^{-1}$ and $44.0 \mathrm{MNm}^{-1}$. The maximum vertical deflection of the rigid roadway pavement at the center of the slab versus the soil stiffness is plotted in Fig. 8. With different values of the soil stiffness, we found several observations. When the stiffness of the soil beneath the slab increased, the vertical displacement at the mid-span of the slab and the maximum flexural stress along the edges of the plate decreased.

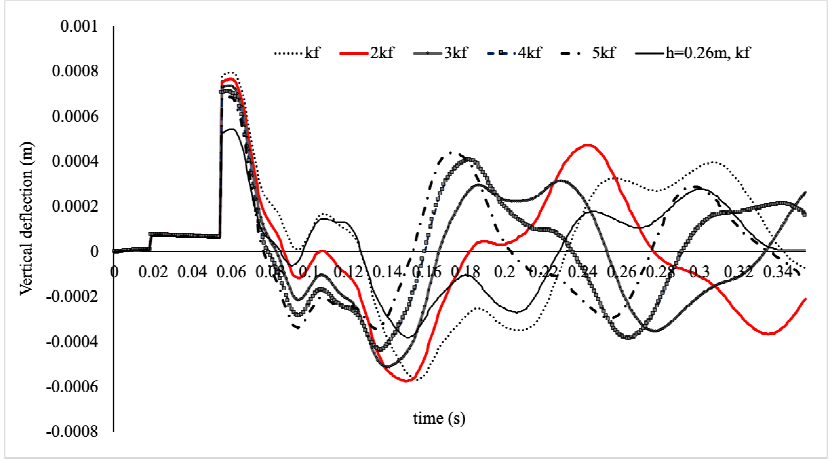

Fig. 8 Vertical displacement at mid-span of the rigid roadway slab versus the soil stiffness $\left(k_{f}\right)$ subjected to a localized blast load

\section{CONCLUSIONS}

In this paper, we numerically analyzed the interaction between a localized blast load and the rigid roadway pavement. The numerical method of a rigid roadway pavement sitting on a two parameters soil foundation model was described by the fourth order partial differential equation where the Levy's problem was used to solve the fundamental frequency of the system. We considered the variation of duration of the positive phase of a blast load, the thickness of the slab and the soil condition beneath the roadway slab to the vertical deflection, vertical shear force along the edge of the slab and the maximum flexural stress within the slab region. The results of this study can be summarized as follow: (a) The ratio of the duration of the blast loading in the positive phase $(t d p)$ over the natural period of the rigid roadway slab $(T n)$ determined the development of maximum response for both the force vibration phases (positive and negative phases) and the free vibration phase; (b) The thickness of the rigid roadway slab greatly affects the overall response of the slabs including the maximum vertical displacement, vertical shear force and the distribution of the flexural stress. The thickness of the slab is important to fulfill the technical requirements of the rigid roadway pavement design and therefore special attention regarding the joint characteristics along the edge of the slab where the dowels and tie-bars are located should be given; (c) Our results show that the stiffness coefficient of soil underneath the slab has a significant influence on the dynamic response of the slab subjected to a localized blast load. By increasing the stiffness coefficient of the soil beneath the slab, the maximum vertical deflection at the mid-span of the slab decreased drastically. However, the rate of decrease depended on the duration of the positive phase of blast load.

In this study, we used a two-parameter soil foundation to analyse slabs with a soil depth of $1.0 \mathrm{~m}-1.5 \mathrm{~m}$. To analyse the slabs with a greater depth, however, a three-parameter soil foundation might be needed to obtain accurate results. Ultimately, taking into accounts blast loads in designing roadway slabs is necessary to account for accurate loads to reduce damage on the structure. In turn, this will help reduce the number of casualties if a terrorist attack occurs.

\section{ACKNOWLEDGMENT}

This research is funded by the Ministry of Research and Technology/National Research and Innovation Agency (RISTEK-BRIN) Republic of Indonesia under grant No. 087/LL3/PG/2020. We gratefully acknowledge the financial support of RISTEK-BRIN. Any opinions, finding and conclusions or recommendations expressed in this paper are those of the authors and do not necessarily reflect the views of RISTEK-BRIN.

\section{REFERENCES}

[1] T. Ngo, P. Mendis, A. Gupta, and J. Ramsay, "Blast Loading and Blast Effects on Structures - An Overview," EJSE: Special Issue: Loading on Structures, pp. 76-81, 2007.

[2] V. Karlos and G. Solomos, "Calculation of Blast Loads for Application to Structural Components," Publications Office of the European Union, Luxembourg, 2013.

[3] G. Lajcakova and J. Melcer, "Numerical Simlation of Moving Load on Concrete Pavements," Transport and Telecommunication, vol. 16, no. 2, pp. 145-157, 2015.

[4] S. W. Alisjahbana, Safrilah, J. C. P. Putra, A. Asmi, I. Alisjahbana, S. Kiryu, and B. S. Gan, "Dynamic Response of Pavement Plates to the Positive and Negative Phases of the Friedlander Load," Strength of Materials, vol. 50, no. 5, pp. 702-710, 2018.

[5] P. Pavel, W. Tanchum and B. Avraham, "Further Modification of Bolotin Method in Vibration Analysis of Rectangular Plates," AIAA Journal, vol. 38, no. 9, pp. 1725-1729, 2000.

[6] B. Souad, K. Salah, and B. Emilio, "Static interaction analysis between beam and layered soil using a two-parameter elastic foundation," International Journal of Advanced Structural Engineering, vol. 11, pp. 21-30, 2019.

[7] S. W. Alisjahbana, I. Alisjahbana, B. S. Gan, and J. C. P. Putra, "Dynamic behaviour of stiffened orthotropic plates subjected to Friedlander blast load," IOP Conf. Series: Materials Science and Engineering, vol. 615, pp. 1-9, 2019.

[8] S. E. Rigby, A. Tyas, T. Bennett, S. D. Clarke, and S. D. Fay, "The Negative Phase of the Blast Load," International Journal of Protective Structures, vol. 5, no. 1-19, 2014. 
[9] S. Susler, H. S. Turkmen, and Z. Kazanci, "The nonlinear dynamic behaviour of tapered laminated plates subjected to blast loading," Shock and Vibration, vol. 19, pp. 1235-1255, 2012.

[10] S. W. Alisjahbana and W. Wangsadinata, "Dynamic analysis of rigid roadway pavement under moving traffic loads with variable velocity,"Interaction and Multiscale Mechanics, vol, 5, no. 2, pp. 105-114, 2012.

[11] S. W. Alisjahbana, I. Alisjahbana, K. Shota, and S. G. Buntara, "Semi analytical Solution of a Rigid Pavement under a Moving Load on a Kerr Foundation Model," Journal of Vibroengineering, vol. 2, no. 5, pp. 2165-2164, 2018 .

[12] S. W. Alisjahbana, A. Asmi, Safrilah, P. Jouvan Chandra Pratama, B. S. G. Gan and I. Alisjahbana, "Numerical simulation of vehicle movement on rigid roadway pavement with discontinuities," Journal of Vibroengineering, vol. 21, no. 5, pp. 1414-1425, 2019.

[13] V. Karlos, G. Solomos, and M. Larcher, " Analysis of the blast wave decay coefficient using the Kingery-Bulmash data," International Journal of Protective Structures, vol. 7, no. 3, pp. 409-429, 2016.

[14] S. E. Rigby, A. Tyas, T. Bennett, S. D. Clarke, and S. D. Fay, "The Negative Phase of the Blast Load," International Journal of Protective Structures, vol. 5, no. 1, pp. 1-19, 2014. A

[15] D. Hrvoje and S. Vladimir, "Blast Loading on Structures," Technical Gazette, vol. 19, no. 3, pp. 643-652, 2012.

[16] H. R. Tavakoli and F. Kiakojouri, "Numerical dynamic analysis of stiffened plates under blast loading," Latin American Journal of Solids and Structures, vol. 11, pp. 185-199, 2014. 